www.jmscr.igmpublication.org

Impact Factor 5.244

Index Copernicus Value: 5.88

ISSN (e)-2347-176x ISSN (p) 2455-0450

crossref DOI:_http://dx.doi.org/10.18535/jmscr/v4i6.58

$\underline{\text { Review Article }}$

\title{
Oxygen Therapy: The Relationship between Individual Characteristics and Effect of Hyperbaric Exposure \\ Authors
}

\section{Albrethen Ibrahim, Albrethen Khalid, Alshehri Abdulhakeem, Alshammari Saleh, Albalawi Ahmad, Alonaizi Naif, Alsaeed Hesham, Bukhari Ahmad}

\section{Introduction}

In their experimental study of subjects who experienced hyperbaric exposure, Risberg, Englund, Aaanderud and Eftedal (2004) suggested need for further investigations to explore the relationship of individual characteristics with effects of hyperbaric exposure. Drawing on articles in the literature, I present here a discursive kind discussion of three topics: 1) the basic physiology of hyperbaric exposure, 2) two major environments of hyperbaric exposure, and 3) certain risks in hyperbaric exposure and some of the population groups implicated.

\section{Hyperbaric Exposure: Physiology}

Put simply, hyperbaric exposure refers to the experience of the pressure of gas at levels above the normal pressure for an atmosphere. The key to understanding the significance of hyperbaric exposure for human beings lies in understanding the physiological implications of this phenomenon as outlined in the literature of Adams (1964), which is the reference point for the next two paragraphs.

The key to the significance of hyperbaric exposure is the impact of the partial pressure of a gas on the human body. Partial pressure is produced when ambient pressure is increased by any percent concentration of a gas. The impact of a gas on the human body is proportional to its partial pressure on the body. When humans breathe $100 \%$ oxygen at sea level/earth's surface, the partial pressure of oxygen is one atmosphere times $20 \%$; this equals 0.2 atmospheres of oxygen. If humans breathe $100 \%$ oxygen at sea level/earth's surface, the partial pressure of oxygen is one atmosphere times $100 \%$; this equals one atmosphere of oxygen. When humans breathe oxygen at four times the pressure that the body experiences at sea level/earth's surface, the partial pressure is 4 atmospheres times 100; this equals 4 atmospheres of oxygen.

Inspired oxygen is carried in the blood as oxyhemoglobin and as dissolved oxygen. Hemoglobin, though important in normal conditions, has limited capacity to transport oxygen. Dissolved oxygen is present in blood plasma, liquid blood, and its volume is limited by the partial pressure of oxygen to which a person is exposed. The more intense the partial pressure of oxygen, the larger the volume of dissolved oxygen. This is the amount of oxygen that the blood releases as it passes through the tissues and returns to the heart. Breathing air at sea level/earth's surface, $0.3 \mathrm{cc}$ of oxygen is dissolved in every 100cc of blood. As pressure rises, the amount of oxygen dissolved increases exponentially. In a hyperbaric exposure 
environment, where $100 \%$ oxygen is inspired at a pressure 4 times greater than at sea level/ earth's surface, $7.2 \mathrm{cc}$ of oxygen is dissolved in each $100 \mathrm{cc}$ of blood. Thus, in the hyperbaric oxygenation environment, the blood gives up much more oxygen than in a normal sea level/earth's surface environment.

\section{Hyperbaric Exposure: Environments}

I focus on two well known hyperbaric exposure environments, underwater diving places and medical clinics.

\section{Diving environment}

According to Bennett, Cronje, and Campbell (2006, pp. 34, 35) diving takes place in an "unnatural environment," where breathing "is done at the expense of the greater partial pressure of the gases [which divers] breathe;" this activity bears "toxicities and risks of effervescence and overexpansion injuries." And "it is only by virtue of special techniques, equipment, procedures, awareness, and knowledge that we are able to survive...the [diving] experience."

Air supply is crucial to function and survival in a diving environment. Naturally, in the logic of the physiology of hyperbaric exposure, divers have to breathe air at pressures which increase exponentially with depth: typically, divers breathe at a pressure of 4 atmospheres absolute. A corresponding effect is the occurrence of an equalisation of pressure in the middle ear spaces, sinuses, and lungs.

All divers are at risk for negative effects of hyperbaric exposure. Their prime hyperbaric exposure risk is twofold: 1) air embolism, particularly venous gas embolism (VGE), a subset of arterial gas embolism, in which gas bubbles form in the systemic venous system; 2) decompression sickness (DCS), which many observers hold as a concomitant condition of VGE. Strauss and Borer (2001) depict air embolism and DCS as characteristic problems of diving activity.

According to the literature of Stoloff, Isenberg and Brill (2001) the pathophysiology of DCS is the development of gas bubbles in the central venous circulation along a pressure gradient. Retention of gas bubbles in the body during diving activity can cause decompression sickness, notably during rapid continuous ascent in the concluding phase of diving. The lethality of these gas bubbles depends to a large degree on their absolute size.

Diagnosis of VGE and DCS, discretely, or combined as cause and effect can be elusive and controversial. Speaking to VGE, Stoloff, Isenberg and Brill (2001, p. 185) observe:

The clinical diagnosis of venous gas embolism may be difficult because of the wide range of symptoms that are similar to those of other acute cardiopulmonary and cerebro-vascular conditions. If small volumes of gas are entrained and symptoms are few, the condition may be unrecognizable. If symptoms are present, they may be ascribed to another etiology.

On DCS, Petri and Andric (2003, pp.26, 27) opine that determination of DCS remains under developed: "we do not have sufficient knowledge to date concerning DCS pathophysiology, onset, and treatment" and "DCS symptoms vary to such a degree from case to case that it has been said that there is no DCS, but merely people who contract it." And Strauss and Borer (2001, p.234) "The correlation of VGE with the symptoms of DCS are [sic] relatively poor. [And] the clearing of Doppler detectable bubbles from the blood stream is not associated with recovery from DCS." These observations imply that divers who succumb to acute VGE are at risk for inaccurate diagnosis, and, in turn, inappropriate treatments.

\section{Medical environment}

In the medical services environment, hyperbaric exposure occurs therapeutically, in hyperbaric medicine, that is hyperbaric oxygen therapy (HBOT), which The Undersea and Hyperbaric Medical Society defines as:

...a treatment, in which a patient breathes $100 \%$ oxygen intermittently while inside a treatment chamber at a pressure higher than sea level pressure (i.e.>1 atmosphere absolute, atm.abs). It can be viewed as the new application of an old, established 
technology to help resolve certain, recalcitrant, expensive, or otherwise hopeless medical problems. In certain circumstances, it represents the primary treatment modality while in others it is an adjunct to surgical or pharmacologic interventions

In an historical note, Leifer (2001) traces a close association between the development of hyperbaric medicine and improvements in diving equipment from the age of Alexander the Great to the current era.

Leifer (2001) cites the list of conditions, for which HBOT is approved by the Undersea and Hyperbaric Medical Society:

- Air embolism

- Thermal burns

- Carbon monoxide and cyanide poisoning

- Traumatic ischemias such as compartment syndrome or crush injuries

- Anemia resulting from exceptional blood loss

- Non healing wounds

- Anaerobic cellulitis or gas gangrene

- Decompression illness ('the bends')

- Acute peripheral arterial insufficiency

- Osteomyelitis

- Radiation tissue damage including osteoradionecrosis

- Actinomycosis

- Intra cranial abscesses

- Compromised skin grafts or flaps HBOT treatment is administered in specially constructed, pressure controlled chambers, of which there are two types: the mono-place chamber, which holds only one patient and does not allow hands-on treatment measures; the multi-place chamber, which can hold up to 12 to 18 patients and equipment for treatments. Both chambers are pressurised with $100 \%$ oxygen. In the multi-place chamber, the patient breathes $100 \%$ oxygen through a hood or mask.

However, HBOT is a controversial field. Chen, Yu, Cheng, Yu and Lo (2006) argue that the safety of HBOT is not conclusive, even for healthy or low risk subjects. The Mayo Clinic (2011) states that evidence is insufficient to support claims that HBOT is an effective treatment for:

- Allergies

- Arthritis

- Autism

- Cancer

- Cerebral palsy

- Chronic fatigue syndrome

- Cirrhosis

- Fibromyalgia

- Gastrointestinal ulcers

- Stroke

Al Waili, Butler, Beale, Mahdi, Finkelstein and Merrow (2006) counsel health professionals and prospective patients that various complications might develop during an HBOT session. Therefore, physicians should be circumspect in prescribing HBOT.

The background to these cautionary counsels is the breathing process in hyperbaric oxygenation circumstances.

\section{Hyperbaric oxygenation and breathing}

In the normal breathing environment of room air, an individual breathes air with a gas composition of $21 \%$ oxygen and $79 \%$ nitrogen. In the breathing process, the following phenomena ensue: the oxygen exchanges for carbon monoxide at the alveolar-capillary membrane, the tissue barrier through which gases are exchanged between the alveolar air and the pulmonary capillaries; the nitrogen content in the residual alveolar air remains relatively stable and is partially responsible for the resiliency of the alveolus. When a person breathes pure oxygen in a hyperbaric exposure environment such as HBOT, the residual nitrogen quickly moves out of the alveolus and is replaced with pure oxygen. Subsequent hyperventilation or obstruction to alveolar ventilation would then favor absorption of that residual oxygen, leading, potentially, to the collapse of the alveolus. Leifer (2001, p. 29) acknowledges the pulmonary risk of hyperbaric oxygenation but, at the same time, notes that duration is a key mediating factor: "Oxygen can be 
toxic to the lungs. After 72 hours of continuously breathing $100 \%$ oxygen at increased atmospheric pressures, the alveolar linings can be damaged."

The toxicity of hyperbaric oxygen derives from the power of free radicals. A free radical is a species of atoms and molecules, which is able to exist independently because its electron is not paired with that of another atom or molecule. De Zwart, Meerman, Commandeur and Vermeulen (1999, p.204) see oxygen derived free radicals as very important mediators of cell injury and cell death, as significant factors in the aging process, and, directly or indirectly, as contributors to various clinical disorders, among them atherosclerosis, reperfusion injury, cancer, brain disorders, lung disorders, and others; oxygen derived free radicals are also important agents in cellular injury consequent on chronic inflammatory processes and central nervous system disorders.

According to Benedetti, Lamorgese, Piersantelli, Pagliarani, Benvenuti and Canestrari, 2003, p.312) production of "increased reactive oxygen species (ROS) in HBOT can cause cellular damage with lipid, protein and DNA oxidation." Reactive oxygen species (ROS) is the collective term used by biologists to include oxygen radicals such as superoxide, alkoxyl, peroxyl, and hydroxyl radicals as well as non radical derivatives from oxygen, hydrogen peroxide and ozone (Ha and Kim, 1999). Speit, Dennog, Radermacher and Rothfuss (2002, p.112) advise that "exposure to oxygen at high ambient pressure can be damaging to mammalian cells" and " an increase in free radicals in the blood from persons undergoing HBOT exposure was directly demonstrated by electron spin resonance (ESR) spectroscopy." ESR is used to measure free radicals presence; it registers the energy changes that take place as unpaired electrons align in response to a magnetic field.

\section{Other HBOT related concerns}

Van Ophoven, Rossbach, Pajank, and Hertle (2005) found that HBOT can cause visual disturbances, eustachion tube dysfunction, and claustrophobia. After their study of the effects of breathing humidified hyperbaric oxygen, on pulmonary function, Shupak, Abramovich, Adir, Goldenberg, Halpern and Ariel (1997, p.246) expressed reservations about this practice, which, according to them, is used routinely in some "HBO therapy protocols." According to Sanders et al. (2012), all HBOT patients are at risk for seizure, especially those with predisposing seizure risk factors: anxiety, fever, hypothermia, brain injury and prior seizure.

In their investigation of the influences of hyperbaric oxygen on patients, Al-Waili et al. (2006) discovered that in HBOT experience, there was a statistically significant elevation of blood pressure and a significant decline in heart rate among patients with diabetes mellitus and hypertension. In their paper on barotraumas, injury due to pressure, Hamilton-Farrell and Bhattacharyya (2004, p.359) identify both diving and HBOT as potential causes of barotraumas, and they note that "artificial ventilation," a feature of both diving and HBOT, may cause pulmonary barotraumas, pressure induced injury to the lungs. This happens, because, in both diving and HBOT, there is "a gas-filled body space, which does not communicate with the environment, and is prevented from equalising pressure or restoring volume in response to an acute change."

Notwithstanding its attribution to various disorders, HBOT has become well incorporated in medical treatment. It should be expected, however, that the medical community will pursue study of its risks and be vigilant for groups which are most vulnerable to these risks. Prominent among these risks is oxidative stress, to which diabetics, smokers and epileptics are main 'at risk' groups

\section{Oxidative Stress}

In Wiernsperger's (2003) literature, oxidative stress (OST) depicts the distortion of a normal physiological phenomenon, the formation of free radicals and reactive oxygen species, by the ineffectual performance of antioxidant systems. Essentially, OST is the result of imbalance between reactive oxygen species and antioxidants; typically, 
this means over production of reactive oxygen species and ineffectiveness of antioxidants. Another view of this occurrence is that it represents an imbalance between pro-oxidative factors and antioxidative factors.

In the scholarship of Moller, Wallin, and Knudsen (1996, p. 18) OST assumes much importance as an agent of disease: "Oxidative stress is a cellular condition implying elevated amounts of...ROS. Today, we encounter the concept of ROS in the elucidations of mechanisms behind several diseases."

\section{Oxidative Stress, Diabetics, and HBOT}

Pazdro and Burgess (2010, p. 282) outline the seriousness of diabetes disease:

It is well established that diabetes not only targets the pancreas, but also causes damage in several other tissues and organs including the kidney, eye, peripheral nerves and brain....With regard to kidney disease, the rise in type 2 diabetes in recent decades has led to it now be [sic] the leading cause of endstage renal failure. Diabetes [also] has profound effects on the nervous system, including both peripheral nerves and the central nervous system.

According to several studies, through its OST effect, hyperbaric exposure, as in diving and HBOT, is a risk for diabetics. Wiernsperger (2003, p. 579) says OST has a "double [negative] impact" on metabolic and vascular functions in diabetics. And Phillips, Cataneo, Cheema, and Greenberg (2004) see OST as a central dynamic in the development of diabetes complications. This dynamic springs from the production of reactive oxygen species, a marked phenomenon of hyperbaric exposure (Pazdro and Burgess, 2010).

Orie, Zidek, and Tepel (1999) said that although the underlying mechanisms of diabetes mellitus (DM) were not completely clear, studies have suggested the involvement of ROS in the pathogenesis and complications of DM. The physiological manifestation of this involvement is the release of superoxide anion, hydroxyl ion, singlet oxygen, and hydrogen peroxide by activated leukocytes.
The outlook for diabetics who seek HBOT is not all pessimistic. According to Zygonis, Garbalosa, Burns, Vidt, and Lowery (2005, p.276) several studies have established that HBOT is an effective treatment for diabetes related wounds; they cite a prospective long- term follow up study of 38 patients with diabetic foot ulcers which documented that HBOT enhanced the pace of healing and minimised the need for amputation.

\section{Oxidative Stress, Smokers, and HBOT}

It is well known that smoking is a major international contributor to various diseases and to mortality. Smokers who apply for diving or HBOT are already at high risk for oxidative stress:

cigarette smoking causes oxidative stress in humans both from the direct effects of the free radicals present in smoke, as well as the inflammatory responses it induces....; smokers are under a high and sustained free radical load, both from cigarette smoke itself and from oxidants produced by activated phagocytes [cells] ( Bruno and Traber, 2006, p. 143).

Smoke related oxidative stress is also a risk for non smokers who experience environmental tobacco smoke, passive smoking in other words. According to Burke and Fitzgerald (2003), passive smoking can cause endothelial dysfunction. Smoking related impaired endothelial function, in turn, implicates the smoking habit in cardiovascular morbidity and mortality.

Smokers who seek HBOT may have low resistance for the OST risks in HBOT, because smoking itself is a force for OST through a sweeping attack on antioxidants, the protective elements against oxidation. In Fearon and Faux's (2009, p.373) observation of this phenomenon: "Cigarette smoking may also down-regulate exogenous and endogenous antioxidants such as vitamin $\mathrm{C}$ (ascorbic acid), carotene, glutathione peroxidise and superoxide dismutase." The assault on antioxidants by cigarette smoking is also explained in the concept of phases of cigarette smoke in the literature of Palanisamy, Bakthavathsalam, Rao and Farook (2009, p.121). They identify two phases of 
cigarette smoke, a tar phase and a gas phase. Both phases, they claim, are "rich in oxygen-centered, carbon-centered, and nitrogen-centered free radicals as well as non-radical oxidants." Smokers can inhale these radicals abundantly in a single cigarette puff; in doing so they ingest compounds which are fertile for the generation of such powerful ROS as superoxide, hydrogen peroxide, hydroxyl and peroxyl radicals, which, in turn, initiate and promote oxidative damage through lipid peroxidation, a putative factor in liver injury.

\section{Oxidative stress, Epileptics, and HBOT}

Chavko, Xing and Keyeser (2001) report that hyperbaric oxygenation yields central nervous system (CNS) toxicity among its adverse consequences, and this, in turn, gives rise to seizure conditions. Leifer (20010 observed that CNS reduces the seizure threshold in patients who are susceptible to seizures. And Shin et al. (2011) argue that oxidative stress in the brain may explain seizure conditions. Coyle and Puttfarcken (1993, p.689) explain the physiology behind this thought. They noted that "the brain consumes a disproportionate amount of the body's [oxygen] as it derives its energy almost exclusively from oxidative metabolism of the mitochondrial respiratory chain." Several structures in the brain together produce a formidable arsenal of oxidants. The mitochondria, for example, are a source of cellular superoxide which is formed during respiration. Against these oxidants, the brain has endogenous antioxidant defences. However, the equilibrium in the brain between ROS derived free radicals and endogenous antioxidant capacity is delicate; it can be disrupted by environments such as hyperbaric oxygenation which intensify oxygen intake during respiration.

Persons with autism spectrum disorders (ASD) are a major group at risk for seizures. In the literature of Matson and Neal (2009) the key manifestations of ASD are communication and social deficits, and issues of comorbid psychopathology and neurodevelopmental disorders. Matson and Neal (2009, p. 1003) also note that "much more needs to be learned" about the "high co-occurrence" of ASD and seizures, and they recommend that clinicians should be mindful of this phenomenon during assessment. This advice is very pertinent to assessment for prospective HBOT.

\section{Conclusion}

The studies that have been outlined in this article underscore that there is room for improvement in knowledge about the role of hyperbaric exposure in some conditions of patients who are under consideration for HBOT. The development of this knowledge would enhance decision making on HBOT application.

\section{References}

1. Adams, J. (1964). Hyperbaric oxygen therapy. The American Journal of Nursing, 64 (6), 76-79. Retrieved 2 June 2012 from http://www.jstor.org/stable/3419100

2. Al-Waili, N., Butler, G., Beale, J., Mahdi, S., Finkelstein, M., Merrow, M. et al. (2006). Influences of hyperbaric oxygen on blood pressure, heart rate, and blood glucose levels in patients with diabetes mellitus and hypertension. Archives of Medical Research, 37 (2006) 991-997. Retrieved 30 May 2012 from Elsevier: Arcmed-D-05-00517

3. Bennett, P., Cronje, F. And Campbell, E. (2006). Assessment of Diving Medical Fitness For Scuba Divers And Instructors. Flagstaff, AZ: Best Publishing Co.

4. Benedetti, S., Lamorgese, A., Piersantelli, M., Pagliarani, S., Benvenuti, F. and Canestrari, F. (2003). Oxidative stress and antioxidant status in patients undergoing prolonged exposure to hyperbaric oxygen. Clinical Biochemistry, 37 (2004), 312-317.

Retrieved 28 May 2012 from www.sciencedirect.com

5. Burke, A. and Fitzgerald, G. (2003). Oxidative stress and smoking-induced vascular injury. Progress in Cardiovascular Diseases, 46 (1), 79-90. Retrieved 2 June 2012 from doi:10.1016/S0033-0620 (03) 00076-8 
6. Bruno, R. and Traber, M. (2006). Vitamin E biokinetics, oxidative stress and cigarette smoking. Pathophysiology 13 (2006), 143149. Retrieved 1 June 2012 from doi:10.1016/j.pathophys.2006.05.003

7. Butler, F. (1995). Diving and hyperbaric ophthalmology. Survey of Ophthalmology, 39 (5), 347-366

8. Chavko, M., Xing, G. and Keyser, D. (2001). Increased sensitivity to seizures in repeated exposures to hyperbaric oxygen: Role of NOS activation. Brain Research, 900 (2001) 227-233. Retrieved 5 June 2012 from Elsevier Science B,V.: PII:S0006/8993 (01) 02301-0

9. Chen, S., Yu, C., Cheng, Y., Yu, S. and Lo, H. (2006). Effects of hyperbaric oxygen therapy on circulating interleukin-8, nitric oxide, and insulin-like growth factors in patients with type 2 diabetes mellitus. Clinical Biochemistry, 40 (2007) 30-36. Retrieved 4 June 2012 from doi:10.1016/j.clinbiochem.2006.07.007

10. Coyle, J. and Puttfarcken, P. (1993). Oxidative stress, glutamate and neurodegenerative disorders. Science New Series, 262 (5134) 689-695 Retrieved 8 June 2012 from http://www.jstor.org/stable/2882673

11. De Zwart, L., Meerman, J., Commandeur, J. and Vermeulen, N. (1999). Free radical biology \& medicine, 26 (1-2), 202-226. Elsevier Science Inc

12. Fearon, I. and Faux, S. (2009). Oxidative stress and cardiovascular disease: Novel tools give (free) radical insight.

Journal of Molecular and Cellular Cardiology, 47 (2009), 372-381. Retrieved 2 June 2012 from Elsevier Inc.: doi:10.1016/ j.jyjmcc.2009.05.013

13. Ha, H. and Kim, K. (1999). Pathogenesis of diabetic nephropathy: The role of oxidative stress and protein kinase $\mathrm{C}$.

Diabetes Research and Clinical Practice, 45 (1999), 147-151. Retrieved 1 June 2012 from Elsevier Science Ltd: PII:S0168-8227 (99) 00044-3

14. Hamilton-Farrell, M. and Bhattacharyya, A. (2004). International Journal of The Care of The Injured, 35, 359-370. Retrieved 30 May 2012 from Elsevier Ltd.: doi:10.1016/j.injury.2003.08.020

15. "Hyperbaric oxygen therapy: Why it's done." Mayo Clinic (27 October 2011). Retrieved 31 May 2012 from http://www.mayoclinic.com

16. "Indications for Hyperbaric Oxygen Therapy." Undersea \& Hyperbaric Medical Society. Retrieved 1 June 2012 from http://memberhsip.pubms.org

17. Leifer, G. (2001). CE credit: Hyperbaric oxygen therapy. The American Journal of Nursing, 101 (8), 26-35. Retrieved 8 June 2012

from

http://www.jstor.org/stable/3522610

18. Matson, J. and Neal, D. (2009). Seizures and epilepsy and their relationship to autism spectrum disorders. Research in Autism Spectrum Disorders, 3 (4) 999-1005 Retrieved 8 June 2012 from http://dx.doi.org/10.1016/j.rasd.2009.06.003

19. Moller, P., Wallin, H. And Knudsen, L. (1996). Oxidative stress associated with exercise, psychological stress and life-style factors. Chemico-Biological Interactions, 102 (1996), 17-36.

Retrieved 2 June 2012 from Elsevier Science Ireland Ltd.: PII S0009-2797 (96) 03729-5

20. Orie, N., Zidek, W. and Tepel, M. (1999). Reactive oxygen species in essential hypertension and non-insulin dependent diabetes mellitus. American Journal of Hypertension Ltd. 12 (12), 1169-1174 Retrieved 9 June 2012 www.ncbi.nlm.nih.gov/pumbmed/10619578

21. Palanisamy, P., Bakthavathsalam, G. Rao, Y. And Farook, J. (2009). Cigarette smoking-Effect of metabolic health risk: A review. Diabetes \& Metabolic Syndrome: 
Clinical Research \& Reviews, 3, 120-127. Retrieved 5 June 2012 from doi:10.1016/jdsx.2009.02.006

22. Pazdro, R. and Burgess, J. (2010). The role of vitamin $\mathrm{E}$ and oxidative stress in diabetes complications. Mechanisms of Ageing and Development, 131 (2010), 276-286.

Retrieved 30 May 2012 from Elsevier Ireland Ltd.: doi:10.1016/j.mad.2010.03.005

23. Petri, N. and Andric, D. (2003). Differential diagnostic problems of decompression sickness-Examples from specialist physicians' practices in diving medicine. Archives of Medical Research 34 (2003), 2630. Retrieved 26 May 2012 from Elsevier Science Inc.: PII S0188-4409 (02) 00458-7

24. Phillips, M., Cataneo, R., Cheema, T. and Greenberg, J. (2004). Increased breath biomarkers of oxidative stress in diabetes mellitus. Clinica Chimica Acta, 344 (2004), 189-194.

Retrieved 30 May 2012 from Elsevier B.V.: doi:10.1016/j.cccn.2004.02.025

25. Risberg, J., Englund, M., Aanderud, L., Eftedal, O., Flook, V. and Thorsen, E. (2004). Venous gas embolism in chamber attendants after hyperbaric exposure. Undersea and Hyperbaric Medical Society, Inc. 31 (4) 417-429

26. Sanders, R., Katz, K., Suyama, J, Akhtar, J., O'Toole, K., Corll, D. et al. (2012). Seizure during hyperbaric oxygen therapy for carbon monoxide toxicity: A case series and five year experience. The Journal of Emergency Medicine, 42 (4), e69-e72.

27. Shin, E., Jeong, J., Chung, Y., Kim, W., Ko, K., Bach, J. et al. (2011). Role of oxidative stress in epileptic seizures. Neurochemistry International, 59 (2), 122-137 Retrieved 7 June 2012 from Elsevier B.V www.ncbi.nlm/nih.gov/pubmed/21672578

28. Shupak, A., Abramovich, A., Adir, Y., Goldenberg, Y., Halpern, P. and Ariel, A. (1997). Effects on pulmonary function of daily exposure to dry or humidified hyperbaric oxygen. Respiration Physiology, 108 (1997) 241-246. Retrieved 7 May 2012 from Elsevier Science: PII S004-5687 (97) 00022-4

29. Speit, G., Dennog, C., Radermacher, P. and Rothfuss, A. (2002). Genotoxicity of hyperbaric oxygen. Mutation Research, 512 (2002), 111-119. Retrieved 31 May 2012 from Elsevier Science B. V.: PII:S13835742 (02) 00045-5

30. Stoloff, D., Isenberg, R. and Brill, A. (2001). Venous air and gas emboli in operative hysteroscopy. The Journal of the American Association of Gynecologic Laparoscopists, 8 (2), 181-192

31. Strauss, M. and Borer, R. (2001). Diving medicine: Contemporary topics and their controversies. American Journal of Emergency Medicine 19 (3), 232-238 Retrieved 2 June 2012 from Doi:10.1053/ajem.2001.22654

32. Van Ophoven, A., Rissbach, G., Pajonk, F. And Hertle, L. (2005). Safety and efficacy of hyperbaric oxygen therapy for the treatment of interstitial cystitis: A randomised, sham controlled, double-blind trial. The Journal of Urology 176, 14421446. Retrieved 27 May 2012 from DOI:10.1016/j.juro.2006.06.065

33. Wiernsperger, N. (2003). Oxidative stress as a therapeutic target in diabetes: revisiting the controversy. Diabetes Metabolism, 29 (6), 579-585

34. Zygonis, T., Garbalosa, J., Burns, P., Vidt, L., and Lowery, C. (2005). A retrospective study of patients with diabetes mellitus after partial foot amputation and hyperbaric oxygen treatment. The Journal of Foot \& Ankle Surgery, 44 (4), 276-280. Retrieved 3 June 2012 from doi: 10.1053/jfas.2005.04.007 